\section{Could ${ }^{18}$ F-FDG PET Be a Diagnostic Tool for Hemochromatosis?}

\section{To the Editor:}

$\mathrm{H}$ ereditary hemochromatosis $(\mathrm{HH})$ is a genetically determined disorder characterized by iron overload. The patients present elevated serum ferritin, high serum iron, and transferrin saturation greater than $45 \%$. If untreated, the pathology evolves to diabetes, heart failure, arthritis, bronze pigmentation of the skin, and liver cirrhosis, which could degenerate in hepatocellular carcinoma. Hereditary hemochromatosis was described in 4 typologies more than 10 years ago. ${ }^{1}$ Type 1 is characterized by mutations in the HFE gene and is the most common genetic disorder with mild severity. The classical variations, which constitute the more frequent genetic background, are either the homozygous mutation p.C282Y or the compound heterozygous mutations C282Y/H63D. Type 2 is the more severe form of the disorder with the onset in the first to third decade of life and is defined as juvenile hemochromatosis. It is characterized by rare mutations in the HAMP (type 2A) or in the HJV (type 2B) genes. ${ }^{2}$ Type 3 has early onset and an intermediate severity and is related to rare mutations in the TFR2 gene. $^{3}$ Type IV is also named ferroportin disease because it has some peculiarities in clinical manifestations and is determined by rare mutations in the SLC4OAI gene. ${ }^{4}$ The penetrance of the more common $\mathrm{HH}$ form (type 1) is incomplete among individuals with genetic predisposition, and variability in the severity of clinical manifestations is often described. Furthermore, not all hemochromatosis cases are classifiable as described above or are explained by mutations in the mentioned genes. The studies of the $\mathrm{HH}$ molecular mechanisms mainly demonstrated the deregulation of the systemic expression of hepcidin or the lack of effect on its receptor ferroportin. The variability of clinical manifestations, which were the object of numerous studies in the past decade, is likely explained by both environmental and genetic factors. ${ }^{5}$

${ }^{18} \mathrm{~F}$-FDG PET is a diagnostic imaging applied in oncology, cardiology, and neurology. The liver and brain have elevated glucose metabolism, and therefore both these organs are characterized by physiologically high ${ }^{18} \mathrm{~F}$-FDG uptake. Inflammation is a pathological condition that augments the glucose metabolism and the ${ }^{18}$ F-FDG uptake; in recent years, its role in inflammatory and infectious disease has been continuously growing.

Recently, we read and appreciated an interesting contribute by Infante et $\mathrm{al}^{6}$ that describes the high liver uptake of ${ }^{18}$ F-FDG on PET/CT in a 64-year-old white man diagnosed with $\mathrm{HH}$. This patient has a history of non-Hodgkin diffuse large B-cell lymphoma treated with chemotherapy, radiotherapy, and stem cell transplant. He underwent ${ }^{18} \mathrm{~F}-\mathrm{FDG}$ PET/CT, and it showed normal distribution. One month later, patient complained abdominal pain and fever. Biochemical parameters showed high level of serum ferritin $(1068 \mathrm{ng} / \mathrm{mL}$; reference range, $10-120 \mathrm{ng} / \mathrm{mL})$. The analysis of $H F E$ gene evidenced in the exon 2 the H63D homozygous mutation. Three months later, a new ${ }^{18} \mathrm{~F}$-FDG PET/CT was performed as a follow-up for his hematological malignancy without biochemical evidences of tumor recurrence. PET/CT images revealed highglucose metabolism in the liver with homogeneous pattern $\left(\mathrm{SUV}_{\max }, 19.1\right)$ and not other pathological finding. After 5 months of follow-up, the patient remained free of disease, and the authors speculated that "increased FDG uptake was not due to tumor disease but to liver damage caused by increased iron deposits probably due to intense hematological therapy." Another case of high liver uptake of ${ }^{18}$ F-FDG in a secondary hemochromatosis case was reported some time ago in this journal. ${ }^{7}$

The high ${ }^{18}$ F-FDG uptake due to iron accumulation could be a very interesting tool in the diagnosis and management of hemochromatosis, which is the most common genetic disorder in white people.

The correlation between iron overload and high ${ }^{18}$ F-FDG liver uptake should deserve further studies in an appropriate cohort of patients affected by hereditary hemochromatosis type 1. Age of onset, sex, and HFE genetic background (C282Y homozigosity or C282Y/H63D compound heterozigosity; H63D homozygous is slightly correlated to hemochromatosis $1 \%$ in males and $0.5 \%$ in females) should be known. Serum ferritin could be elevated as a consequence of other factors such as acute or chronic inflammation, cancer, hepatitis, and hyperferritinemia cataract syndrome in addition to iron overload. Therefore, additional parameters of iron overload (serum iron and transferrin saturation) should be necessary to study hemochromatosis. Biochemical parameters of hepatic functionality should be dosed to complete the clinical hepatic functional profile. The patients should undergo ${ }^{18} \mathrm{~F}$-FDG PET/CT, and liver uptake should be correlated with biochemical parameters of iron overload and with the amount of the liver iron concentration evaluated by liver biopsy. If ${ }^{18}$ F-FDG uptake depends on iron overload, scientists could elaborate a mathematical model to define the correlation. To complete the studies, a new follow-up ${ }^{18}$ F-FDG PET/ CT should be performed to verify normalization of the liver uptake after the required phlebotomies and the normalization of the iron parameters. The evidences of correlation between iron concentration and high

${ }^{18}$ F-FDG uptake could have important implications also on neurodegenerative disease characterized by iron accumulation in the specific brain areas such as NBIA, Parkinson disease, Alzheimer disease, Huntington disease, and multiple sclerosis.

In conclusion, we compliment with the authors for the important observation of high ${ }^{18} \mathrm{~F}$-FDG liver uptake in a patient with $\mathrm{HH}$ opening a new door to a possible important tool for the diagnosis of this pathology.

\section{ACKNOWLEDGMENTS}

The authors thank God for enabling them to work for people who are ill.

Isabella Zanella, PhD

Department of Molecular and Translational Medicine University of Brescia and Biotechnology/3rd Laboratory Department of Diagnostics Civic Hospital of Brescia Brescia, Italy

Francesco Bertagna, MD

Department of Nuclear Medicine University of Brescia and Spedali Civili of Brescia Brescia, Italy

Giorgio Biasiotto, PhD

Department of Molecular and Translational

Medicine, University of Brescia and Biotechnology/3rd Laboratory

Department of Diagnostics Civic Hospital of Brescia Brescia, Italy

giorgio.biasiotto@unibs.it

Conflicts of interest and sources of funding: none declared.

\section{REFERENCES}

1. Pietrangelo A. Hereditary hemochromatosis new look at an old disease. $N$ Engl J Med. 2004; 350:2383-2397. 
2. Camaschella C, Roetto A, De Gobbi M. Juvenile hemochromatosis. Semin Hematol. 2002; 39:242-248.

3. Biasiotto G, Camaschella C, Forni GL, et al. New TFR2 mutations in young Italian patients with hemochromatosis. Haematologica. 2008; 93:309-310.
4. Pietrangelo A. The ferroportin disease. Blood Cells Mol Dis. 2004;32:131-138.

5. Zanella I, Rossini A, Di Lorenzo D, et al. Hereditary hemochromatosis: the same old song. Blood Cells Mol Dis. 2015;55:216-217.

6. Infante JR, Moreno M, Rayo JI, et al. High liver FDG uptake on $\mathrm{PET} / \mathrm{CT}$ in patient with lymphoma diagnosed with hereditary hemochromatosis. Clin Nucl Med. 2015;40: 538-539.

7. Ito K, Minamimoto R, Morooka M, et al. A case of secondary hemochromatosis with high uptake of liver in F-18 FDG PET/CT imaging. Clin Nucl Med. 2011;36:606-608. 